MIS, Vol. 5, No. 2, July - December 2006, pp. 1-13

ISSN 0975-3311

https:/ / doi.org/ 10.12725/ mjs.9.1

\title{
DARCY-BÉNARD CONVECTION USING A NON-FOURIER THERMAL MODEL
}

\author{
P. G. Siddheshwar*
}

\begin{abstract}
In the paper the effect of replacing the classical Fourier thermal model by the non-classical non-Fourier thermal model on Darcy-Benard convection in a fluid-saturated densely-packed porous medium is studied and, in particular, how the Nusselt numbers of the solid and fluid phases are affected by the adoption of such a model is considered. A weightedoverage Nusselt number is also defined and analyzed to study the overall heat transfer in the case of steady finite-amplitude convection which is omenable to analytical treatment. Sub-critical instability is ruled out in mono-diffusive convection but the inclusion of a seconddiffusing component or rotation is shown to facilitate its manifestation.
\end{abstract}

\section{Introduction}

Darcy-Bénard convection in a porous medium is a phenomenon relevant to many fields in geophysical, environmental and technological applications. The state-ofart on the subject has been well documented in the books of Nield and Bejan[1], Vafai[2] and Ingham and Pop [3]. Most studies concerning the problem have

* Royal Sociefy Incoming Fellow, Department of Mechanical Engineering, University of Both, BA2 7AY, UK, pgsiddeshwar@hotmail.com.

(Permanent Address : Department of Mathematics, Bangalore University, Bangalore 560001) 
assumed that the convecting liquid and the porous medium are everywhere in local thermal equilibrium (LTE). Nield and Bejan[1] have advocated the need to have a two-equation model to study convection in porous media. In the two-field or two-equation model the energy equations of the fluid and solid phases are coupled by means of terms which account for the heat lost to or gained from the other phase. Quintard et al.[4] describes this as the simplest form that reflects the concept of two macroscopic media. Thus the inter-phase heat transfer is modeled by a heat transfer coefficient, $h$, whose value depends on the nature of the porous matrix and the saturating fluid. In a review article Vafai and Amiri[5] discuss possible values of this coefficient which has been the subject of intense experimental interest. Large values of $h$ correspond to a rapid transfer of heat between the phases, and therefore such values are taken to correspond to LTE. Conversely, small values of $h$ give rise to relatively strong non-equilibrium effects. The two-field model is now being used extensively, especially in the realm of forced convective transport, and the works of Nield and Kuznetsov[6], Calmidi and Mahajan[7] and Carbonell and Whitaker[8] discuss typical situations which require the use of the model. Sparse literature available on the Darcy-Bénard convection problem using a two-field model (see [9] and [10]) concentrate on the onset problem. A global nonlinear stability of the problem has been performed by Straughan [11]. Very recently, Sheu[12] has solved the Lorenz model for chaotic convection in a porous medium using a LTNE model. The aim of the present paper is to present the classical Darcy-Bénard problem in a new perspective by replacing the classical Fourier thermal model(FTM) with the non-classical non-Fourier thermal model(NFTM) and seeing its effect on aspects associated with the nonlinear realm of convection, especially on the Nusselt numbers of the fluid and solid phases, and also on the weighted-average Nusselt number. Specifically the following is aimed at in the paper:

i) Present the Darcy-Bénard convection problem in a new perspective and ascertain mathematically the reason behind the delay in the onset of convection in the NFTM compared to the FTM.

ii) Verify if the neglection of the local acceleration term is justified in the study of convection using the NFTM. In other words, check whether the principle of exchange of stabilities (PES) is valid or not when the local acceleration term is present.

iii) Delineate the separate contributions of the solid and fluid phases to the overall heat transport in the porous medium.

iv) Investigate the possibility of subcritical motions (both steady and oscillatory) in each of mono-diffusive, double-diffusive and rotating systems. 


\section{Mathematical Formulation}

Consider a horizontal isotropic porous layer of infinite extent occupied by a Boussinesq fluid, confined between stress-free, isothermal boundaries at $y=0$ and $y=d$, at which the fluid temperatures are $T_{1}=T_{0}+\Delta T$ and $T_{u}=T_{0}$ respectively (see figure 1). The medium is assumed to have low-porosity and hence the fluid flow is governed by Darcy model. We consider Darcy-Bénard convection in this layer of depth, $d$. The basic state is assumed to be quiescent with the conduction temperature profiles of both fluid and solid phases having the non-dimensional

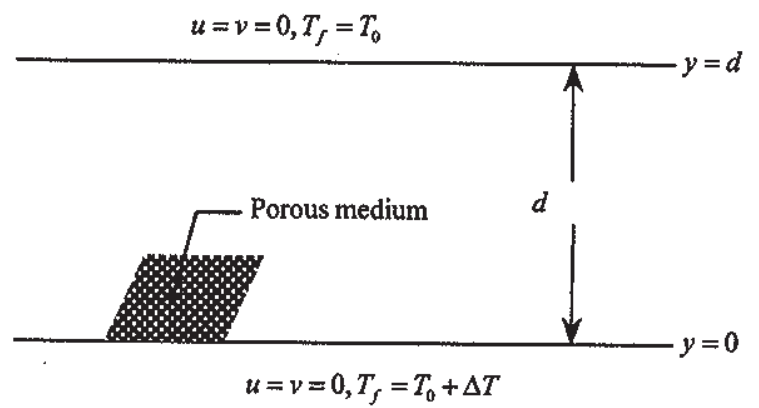

Figure 1. Definition sketch of the horizontal porous layer and boundary conditions.

distribution $]-y$. The governing perturbation equations for the problem in nondimensional form are (Siddheshwar [13]):

$$
\begin{aligned}
& U_{x}+V_{y}=0 \\
& \left(\frac{1}{V a_{D}} \frac{\partial}{\partial t}+1\right)\left(V_{x x}+V_{y y}\right)=R \Theta_{x x \prime} \\
& C_{p v} \frac{\partial^{2} \Theta}{\partial t^{2}}+\frac{\partial \Theta}{\partial t}+\left(1+C_{D N} \frac{\partial}{\partial t}-\frac{\sec \xi}{H^{9}} \nabla^{2}\right)\left[U \Theta_{x}+V \Theta_{y}+V\right] \\
& =\left(1+C_{N v} \frac{\partial}{\partial t}-\frac{\sec \xi}{H^{2}(1+\tan \xi)} \nabla^{2}\right) \nabla^{2} \Theta
\end{aligned}
$$


where

$$
\begin{aligned}
& V_{D}=\frac{\mu(\rho c)_{f} k}{\rho_{f} k_{f} d^{2}} \\
& \text { (Nadasz number or Prandtl-Darcy number), } \\
& R=\frac{\rho_{f} g \beta\left(T_{1}-T_{v}\right) K d}{\mu \varepsilon k_{i}(T+\tan \xi)} \quad \text { (Darcy-Rayleigh number), } \\
& \alpha=\frac{(\rho c)_{s}}{(\rho c)_{f}} \frac{k_{f}}{k_{s}}=\frac{\kappa_{f}}{\kappa_{s}} \quad \text { (porosity-modified conductivity ratio), } \\
& C_{p y}=\frac{\alpha}{H^{*}}\left[\frac{1+\tan \xi}{(1+\alpha \tan \xi)^{2}}\right] \quad \text { (Cattaneo-Vadasz number), } \\
& C_{D N}=\frac{\alpha}{H^{\prime} \cos \xi}\left[\frac{1+\tan \xi}{1+\alpha \tan \xi}\right] \text { (Cattaneo-Nield number), }
\end{aligned}
$$

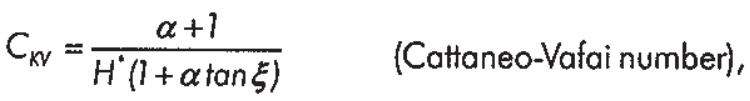

$$
\begin{aligned}
& \left(H^{*}, \xi\right)=\left(d^{2} h \sqrt{\frac{1}{\varepsilon^{2} k_{f}^{2}}+\frac{1}{(1-\varepsilon)^{2} k_{s}^{2}}}, \tan ^{-1}\left[\frac{(I-\varepsilon) k_{s}}{d k_{f}}\right]\right) \text { are the Rees numbers. }
\end{aligned}
$$

The boundary conditions for solving (1)-(3) are

$$
\begin{array}{ll}
V=\Theta=\Theta_{w}=0 & \text { on } y=0, \\
V=\Theta=\Theta_{y}=0 & \text { on } y=1 .
\end{array}
$$

It suffices to use just Eq. (2) with Eq.(3) to determine the onset Darcy-Rayleigh number. An equation for the solid phases' temperature is, however, required to determine the heat transport in the solid phase.

The great advantage of the NFTM in the present form is the ease it provides in switching over to the FTM through a unique limiting procedure $H^{*} \rightarrow \infty$. In the 
event of the Rees numbers not being used in the formulation then the switch over could be effected in two different ways(see Banu and Rees[]). Further, Rees numbers provide symmetry of representation as is evident from their expression.

The equations have been rendered non-dimensional using $d$ for spatial coordinates, $\frac{\left[\varepsilon(\rho c)_{f}+(1-\varepsilon)(\rho c)_{s}\right] d^{2}}{\varepsilon k_{i}+(I-\varepsilon) k_{s}}$ for time, $\frac{\varepsilon k_{f}+(I-\varepsilon) k_{s}}{(\rho c)_{i} d}$ for velocity and $\left(T_{1}-T_{u}\right)$ for temperature of both the fluid and solid phases. In the above equations $U$ and $V$ are the fluid velocity components in the horizontal and vertical directions, respectively, $x$ and $y$ are the corresponding Cartesian coordinates and $t$ is time. The following are the other fluid and medium properties: $K$ is the permeability, $\mu$ the fluid viscosity, $\rho$ the density, $c$ the specific heat, $\beta$ the coefficient of thermal expansion, $\varepsilon$ the porosity and $k$ the thermal conductivity. The subscripts $s$ and $f$ denote the quantities of the solid and fluid phases, respectively. The time derivative in Eq. (2) has been retained to verify if the adoption of NFTM leads to the principle of exchange of stabilities being violated. Instinct drives one to think of oscillatory convection being possible in Darcy-Bénard convection with NFTM due to the presence of a second order derivative in time.

Equations (1)-(3), subject to the boundary conditions (4), admit a minimal Fourier solution in the form:

$$
\left[\begin{array}{l}
U \\
V \\
\Theta
\end{array}\right]=\left[\begin{array}{cc}
-\frac{\sqrt{8 \pi} A_{H} \pi \delta \sqrt{r-1}}{k} A(t) \sin k x \cos \pi y \\
\sqrt{8 \pi} A_{t} \delta \sqrt{r-1} A(t) \cos k x \sin \pi y \\
-\frac{\sqrt{8 \pi}}{\delta} \frac{r-1}{r} B(t) \sin k x \sin \pi y
\end{array}\right]-\left[\begin{array}{l}
0 \\
0 \\
\frac{1}{\pi} \frac{r-1}{r} D(t)
\end{array}\right] \sin 2 \pi y, \quad(5 a, b, c)
$$

where $k$ is the horizontal wave number, $\Delta_{H}=\frac{\Delta_{2}}{\Delta_{1}}, \Delta_{4}=1+\frac{\delta^{2} \sec \xi}{H^{*}}$ and, $\Delta_{2}=1+\frac{\delta^{2} \sec \xi}{H^{2}(1+\tan \xi)}, r=\frac{R k^{2}}{\delta^{4} \Delta_{t}}$ and $\delta^{2}=\pi^{2}+k^{2}$. Equation (5) is a roll-solution which is assumed with the knowledge that rolls remain the favoured pattern for all combination of values of the Rees numbers (see Rees and Pop [14]).

Substitution of (5) into (2) and (3) yields the following generalized Vadasz-Lorenz model for nonlinear convection in porous media: 


$$
\begin{aligned}
& \left(1+\frac{1}{V a_{D}^{0}} \frac{d}{d \tau}\right) A=B \\
& \left(1+C_{P v}^{\cdot} \frac{d}{d \tau}\right) \frac{d B}{d \tau}=r \Delta_{H}\left(\Delta_{1}+C_{D N}^{\cdot} \frac{d}{d \tau}\right) A-\Delta_{H}(r-1)\left(\Delta_{1}+C_{D N}^{\cdot} \frac{d}{d \tau}\right) A D \\
& -\left(\Delta_{2}+C_{k v} \frac{d}{d \tau}\right) B \text {, } \\
& \left(1+C_{P v}^{*} \frac{d}{d \tau}\right) \frac{d D}{d \tau}=4 \gamma^{2} \Delta_{H}\left(\Delta_{1}+C_{D N}^{*} \frac{d}{d \tau}\right) A B-4 \gamma^{2}\left(\Delta_{2}+C_{K v}^{*} \frac{d}{d \tau}\right) D_{1}
\end{aligned}
$$

where

$$
\tau=\delta^{2} t, V a_{D}^{\cdot}=\frac{V a_{D}}{\delta^{2}}, C_{P V}^{\cdot}=C_{p V} \delta^{2}, C_{D N}^{*}=C_{D N} \delta^{2}, C_{k V}^{\cdot}=C_{K V} \delta^{2} \text { and } \gamma^{2}=\frac{\pi^{2}}{\delta^{2}}
$$

One can easily verify that Eqs. (6)-(8) reduce to the Vadasz-Lorenz model of DarcyBénard convection using FTM, reported by Vadasz[15], in the limit $H^{*} \rightarrow \infty$. A Lorenz model for anisotropic porous media convection was derived and analysed by Kakimoto et al.[16] Various aspects of nonlinear convection in porous media was reviewed by Rudraiah et al.[17]. The expression for the onset Rayleigh number is presented next followed by aspects of the nonlinear realm.

Assuming the amplitudes $A$ and $B$ to have the form $e^{\text {iot }}$, the linear version of Eqs. (6) and (7) yields the Darcy-Rayleigh number expression to determine the onset of convection and is given by:

$$
r=\frac{\Delta_{1} \Delta_{2}-\Delta_{1} \omega^{2}\left(C_{p V}^{*}+\frac{\left(1+C_{R V}^{*}\right)}{V_{\sigma_{D}^{*}}^{*}}\right)-\left[C_{D N}^{*}\left[1+C_{k V}^{*}\right]-\frac{\Delta_{2}-C_{p V}^{*} \omega^{2}}{V_{a_{D}^{*}}^{*}}\right] \omega^{2}}{\Delta_{H}\left(\Delta_{1}^{2}+C_{D N}^{+2} \omega^{2}\right)}+i \omega N,
$$

where

$$
N=\frac{\Delta_{1}\left[1+C_{k V}^{*}+\frac{\Delta_{2}-C_{p V}^{*} \omega^{2}}{V \sigma_{0}^{*}}\right]-\left[C_{D N}^{\cdot}\left[\Delta_{2}-\omega^{2} C_{p V}^{*}\right]-\frac{\left(1+C_{k V}^{*}\right)}{V \sigma_{0}^{*}} \omega^{2}\right]}{\Delta_{H}\left(\Delta_{1}^{2}+C_{D N}^{*} \omega^{2}\right)}
$$


Since $r$ is a real quantity, either $\omega=0$ or $N=0$ (with $\omega \neq 0$ ). The former and latter conditions refer respectively to stationary and oscillatory convections. The latter condition yields an expression for $\omega$ in the form:

$$
\omega^{2}=\Delta_{1} \frac{\left[\left(1+C_{K V}^{*}\right)-C_{D N}^{*} \frac{\Delta_{2}}{\Delta_{1}}+\frac{\Delta_{2}}{V a_{D}^{*}}\right]}{\left[\frac{\Delta_{1} C_{P V}^{*}-\left(1+C_{K V}^{*}\right)}{V a_{D}}\right]-C_{D N}^{*} C_{P V}^{*}},
$$

and this is not positive for any combination of the parameter values. The inference thus is that the principle of exchange of stabilities is valid in the Darcy-Bénard problem even in the case when the thermal model is non-Fourier and the local acceleration is non-zero in the Darcy law of Eq. (2). This is in concurrence with the findings of Banu and Rees[9].

The stationary Rayleigh number for the onset of convection is given by $r=1$. Reverting back to unscaled quantities using the definitions given earlier, this condition translates to:

$$
R=\frac{\delta^{4}}{k^{2}}\left[1+\frac{H^{*} \sin \xi}{\delta^{2}+H^{*} \cos \xi}\right] \frac{1}{1+\tan \xi} .
$$

The form of equation (13) is intentional to indicate explicitly the contribution of NFTM to the onset of convection. It is obvious from the equation that

$$
R^{N F T M}=R^{F T M}\left[1+\frac{H^{*} \sin \xi}{\delta^{2}+H^{*} \cos \xi}\right] \frac{1}{1+\tan \xi^{\prime}}
$$

where the superscripts NFTM and FTM on $R$ denote the acronyms used earlier. The critical wave number $k_{\mathrm{C}}$ that determines the smallest $R_{0}=R(l+\tan \xi)$, viz., $R_{\mathrm{DC}}$ that signals the onset of convection, is the smallest positive root of the equation

$$
\begin{aligned}
\left(k^{2}\right)^{3}+\left(\pi^{2}+2 H^{\prime} \cos \xi\right)\left(k^{2}\right)^{2} & \\
& +\left[H^{-2} \cos \xi(\cos \xi+\sin \xi)-\pi^{2} H^{2} \sin \xi-\pi^{4}\right] k^{2} \\
& \quad-\pi^{2}\left(\pi^{2}+H^{4} \cos \xi\right)\left[\pi^{2}+H^{2}(\cos \xi+\sin \xi)\right]=0 .
\end{aligned}
$$


In the limit $H^{*} \rightarrow \infty$, it can easily be seen from Eqs. (14) and (15) respectively that

$$
R_{D}^{\text {NFTM }} \rightarrow R^{\text {FTM }} \text { and } k_{C} \rightarrow \pi
$$

where $k_{c}$ is the critical wave number. Further, it is pretty obvious that $R_{0}^{\text {NFTM }}>R^{\text {FTM }}$, in general.

The non-linear system of autonomous differential equations (6)-(8) is not analytically tractable for the general time-dependent variables and will have to be solved using a numerical procedure. The steady finite-amplitude system is, however, amenable to analytical treatment and one may obtain $B$ and $C$ in terms of $A$, and their expressions are recorded below:

$$
B=A, \quad D=\frac{r \Delta_{H} \Delta_{1}+\Delta_{2}}{\Delta_{H} \Delta_{3}(r-1)}
$$

where $A$ is given by

$$
A^{2}=\frac{\Delta_{2}}{\Delta_{H}^{2} \Delta_{3}^{2}} \frac{r \Delta_{1} \Delta_{1}+\Delta_{2}}{(r-1)}
$$

In the succeeding section we delineate the individual contributions of the fluid and solid phases to the overall heat transport by steady convection in a porous medium.

\section{Heat Transport}

In the study of convection problems the determination of heat transport across the fluid layer is important. This is because the onset of convection as Rayleigh number is increased is more readily detected by its effect on the heat transfer. In the basic state, the heat transfer is by conduction alone.

The fluid Nusselt number, $N u_{f \prime}$ is given by

$$
N u_{f}=\frac{\left[\frac{k}{2 \pi} \int_{0}^{2 \pi / k}(1-y+\Theta)_{y} d x\right]_{y=0}}{\left[\frac{k}{2 \pi} \int_{0}^{2 \pi / k}(1-y)_{y} d x\right]_{y=0}}
$$


The term $1-y$ in the integrand is the dimensionless temperature distribution of conduction state prevalent before convection sets in. The second term represents the convective contribution to heat transport. Substitution of Eq. (5c) in Eq. (19), and completion of integration yields

$$
N u_{f}=1+2\left[\frac{r-1}{r}\right] D
$$

We now obtain the expression of the Nusselt number for steady convection by the substitution of the expression of $D$ from Eqs.(17) into Eq. (20). This yields $\mathrm{Nu}$ in the form:

$$
N u_{f}=1+2\left[1-\frac{1}{r}\right]
$$

The solid Nusselt number, $\mathrm{Nu}_{\mathrm{s}^{\prime}}$ is given by

$$
N U_{s}=\frac{\left[\frac{k}{2 \pi} \int_{0}^{2 \pi / k}(1-y+\Phi)_{y} d x\right]_{y=0}}{\left[\frac{k}{2 \pi} \int_{0}^{2 \pi / k}(l-y)_{y} d x\right]_{y=0}}
$$

where $\Phi$ is the temperature of the solid phase that enjoys exactly the same form as Eq. (3) for $\Theta$ and the minimal representation for $\Phi$ is the same as that in Eq. (5) for $\Theta$ but with the amplitude of the linear and nonlinear modes now being $C$ and $E$ respectively. Substitution of the minimal representation of $\Phi$ in Eq. (22) and expressing $E$ in terms of $A$ yields the following form for $\mathrm{Nu}_{s}$ in the case of steady convection:

$$
\left(N u_{s}-1\right)=\frac{H^{*} \cos \xi}{4 \pi^{2}+H^{*} \cdot \cos \xi}\left(N u_{f}-1\right)
$$

It is obvious from the above equation that $\mathrm{Nu}_{s} \leq N u_{f}$, with the equality being true when $H^{*}$ is very large.

A discussion on the overall heat transfer in the porous medium can be made by analyzing a weighted-average Nusselt number $\mathrm{Nu}_{w}$ involving $\mathrm{Nu}$, and $\mathrm{Nu}$. So we define $\mathrm{Nu}_{w}$ as: 


$$
N u_{w}=\frac{N u_{f} \cos \xi+N u_{s} \sin \xi}{\cos \xi+\sin \xi}=1+2 \frac{\left[\frac{4 \pi^{2}}{1+\tan \xi}+H^{\circ} \cos \xi\right]}{\left[4 \pi^{2}+H^{\circ} \cos \xi\right]}\left(1-\frac{1}{r}\right) .
$$

It is obvious from the above equation that

$$
N u^{N F T M}<N u^{\text {FTM }},
$$

where $N u^{N F T M}$ is essentially $N u_{w}$ and $N u^{\text {NFTM }}=N u_{f}=N u_{s}$. In the succeeding section we look into the possibility of sub-critical instability.

\section{Sub-critical instability}

To explore the possibility of sub-critical motions, we expand $A, B, D, \omega$ and $r$ in a series involving $\varepsilon$ that gets identified with the amplitude of finite-amplitude motions in the course of the analysis.

$$
\begin{aligned}
& A=\varepsilon A_{1}+\varepsilon^{3} A_{3}+\ldots \ldots \ldots \\
& B=\varepsilon B_{1}+\varepsilon^{3} B_{3}+\ldots \ldots \ldots . \\
& D=\varepsilon^{2} D_{2}+\varepsilon^{3} D_{3}+\ldots \ldots \ldots \\
& \omega=\omega_{0}+\varepsilon^{2} \omega_{2}+\varepsilon^{3} \omega_{3}+\ldots \ldots \ldots . . \\
& r=r_{0}+\varepsilon^{2} r_{2}+\varepsilon^{3} r_{3}+\ldots \ldots \ldots . .
\end{aligned}
$$

Since the principle of exchange of stabilities is valid, we note that $\omega_{0}=0, r_{0}=1$ that in turn implies $R_{\mathrm{o}}=\frac{\delta^{4} \Delta_{H}}{k^{2}}$. Substituting equation (26) in equations (6)-(8), and equating coefficients of various powers of $\varepsilon$, we gef

$$
\begin{aligned}
& \left\lfloor\left[A_{1}, B_{1}\right]^{T}=[0,0]^{T},\right. \\
& \left\lfloor\left[A_{3}, B_{3}\right]^{T}=\left[(\ldots) \omega_{2},(\ldots) \omega_{2}+(\ldots)+(\ldots) r_{2}\right]^{T},\right.
\end{aligned}
$$


and so on. In the above equations, $L$ has the following definition:

$$
L=\left[\begin{array}{ll}
7 & -1 \\
\Delta_{H} \Delta_{1} r_{O} & -\Delta_{2}
\end{array}\right],
$$

and the parantheses (...) on the right hand side of equation (28) are expressions involving the parameters of the problem. The condition for the existence of a nontrivial solution to equation (27) requires $L$ to be singular. Equation (28) clearly implies that $\omega_{2}=0$ and $r_{2}=(\ldots)\left(7-r_{0}\right)$. Since $r_{0}=7$, due to the principle of exchange of stabilities being valid, $r_{2}$ is also zero. One may proceed to higher order corrections but in the problem these make no further contribution to the frequency and Rayleigh number. In other words there is no possibility of sub-critical motions in convection in densely-packed porous media using the non-Fourier thermal model. This is true of traveling wave instability as well.

\section{Results and Discussion}

1. Darcy-Bénard convection problem is seen in a new perspective using the 'hyperbolic-type' of heat equation and the simplicity of such a usage. The reason behind the delay in the onset of convection in the NFTM model, compared to the FTM model, is the 'hyperbolic-type' of equation (with biharmonic correction) enjoyed by the temperatures of the solid and fluid phases.

2. The neglection of the local acceleration term is justified in the study of convection using the NFTM model. PES is valid when the local acceleration term is present and the NFTM is adopted.

3. The separate contributions of the solid and fluid phases to the overall heat transport in the porous medium is delineated:

4. Sub-critical motions are shown not to manifest in mono-diffusive convection in porous media with both NFTM and FTM models. However, the presence of a second diffusing component gives rise to not only the possibility of oscillatory convection but also sub-critical motions. This is true of rotating systems as well. The onset thermal Rayleigh number and the frequency of oscillations in the case of the double-diffusive convection are respectively given by:

$$
R a_{T}=J \delta^{2} \frac{\left[\delta^{2}\left(1+\delta^{2} C_{K V}\right)+\left(\delta^{2} \Delta_{2}-\omega^{2} C_{P V}\right) L e \varepsilon\right]}{a^{2}\left[C_{D N} \delta^{2}+\Delta_{\mathrm{l}} L e \varepsilon+N L e\left(1+\delta^{2} C_{K V}\right)\right]},
$$




$$
\delta_{4} C_{\rho v} L e \varepsilon\left(\omega^{2}\right)^{2}+\left(\delta_{2} \delta_{6}-\delta_{3} C_{\rho v} L e \varepsilon-\delta_{4} \delta_{5}\right) \omega^{2}-\delta_{1} \delta_{6}=0
$$

and $J=1$ refers to the porous medium heated by below where the highest concentration is imposed and $J=-1$ to heating from the top where the highest concentration is imposed (Mojtabi and Charrier-Mojtabi[18]). The quantities $L e, \varepsilon$ and $N$ are the Lewis number, porosity and the buoyancyratio respectively. The $\delta_{i}$ 's are functions of the parameters of the problem and are not recorded here. The finite amplitude Rayleigh number, $R_{\mu}$ is given by:

$$
k^{4} L e^{2}(\Delta+N) R_{f}^{2}+2 \delta^{4} J k^{2} L e(N-\Delta)\left(I-\Delta^{2} L e\right) R_{f}+\delta^{8}\left(I-\Delta^{2} L e\right)^{2}=0,
$$

where $\Delta=\frac{\Delta_{2}}{\Delta_{1}}$ and the other quantities have their earlier meaning. It was found that oscillatory and sub-critcal stationary-wave instabilities are possible in this case. In the limit of $\mathrm{H}^{*} \rightarrow \infty$, the results of Mojtabi and CharrierMojtabi[18] are recovered.

\section{Acknowledgement}

The author would like to thank the Royal Society, London for awarding him a visiting fellowship for a year. This work was completed after several discussions with Dr. Andrew Rees and while enjoying his hospitality. The author is grateful to the University of Bath for hosting him and also to the Bangalore University, India for granting him leave.

\section{References}

1. D. A. Nield, A. Bejan, Convection in Porous Media, Third ed., Springer, Berlin, 2006.

2. K. Vofai, Handbook of Porous Media, Marcel Dekker, 2000.

3. D. B. Ingham, I. Pop (Eds.), Transport Phenomena in Porous Media, Pergamon, Oxford, 1998.

4. M. Quintard, Modelling local non-equilibrium heat transfer in porous media, in: Proc. $11^{\text {th }}$ Int. Heat Transfer Conf., Kyong-ju, Korea, August 1998, vol. 1,1998, pp. 279-285.

5. K. Vafai, A. Amiri, Non-Darcian effects in confined forced convective flows, in: D. B. Ingham, I. Pop (Eds.), Transport Phenomena in Porous Media, Pergamon Press, Oxford, 1998, pp. 313-329. 
6. D. Nield, A.V. Kuznetsov, The interaction of thermal non-equilibrium and heterogeneous conductivity effects in forced convection in layered porous channels, Int. J. Heat Mass Transfer, vol.44, 2001, pp. 4369-4373.

7. V. V. Calmidi, R. L. Mahajan, Forced convection in high porosity foams, Trans. ASME J. Heot Transfer, vol.122, 2000, pp. 557-565.

8. R. G. Carbonell, S. Whitaker, Heat and mass transfer in porous media, in: J. Bear, M.Y. Carapcioglu (Eds.), Fundamentals of Transport Phenomena in Porous Media, NATO ASI Series E: Applied Sciences, Barking, vol.82, 1982, pp. 123-198.

9. N. Banu, D. A. S. Rees; Onset of Darcy-Benard convection using a thermal non-equilibrium model, Int. J. Heat and Mass Transfer, vol. 45, 2002, pp. 2221-2228.

10. A. Postelnicu, D. A. S. Rees, Onset of Darcy-Brinkman convection using a thermal nonequilibrium model-part I: stress-free boundaries, Int. J. Energy Res., vol. 27, 2003, pp. 961-973.

11. B. Straughan, Global stability in porous convection with a thermal non-equilibrium model, Proc. Roy. Soc. A, London, vol. 462, 2005, 409.418.

12. L. J. Sheu, An autonomous system for chaotic convection in a porous medium using a thermal non-equilibrium model, Chaos, Solitons and Fractals, vol. 30, 2006, pp. 672-689.

13. P. G. Siddheshwar, A new perspective on Darcy-Bénard convection using a non-Fourier thermal model, Phys. of Fluids, 2006 (to be submitted).

14. D. A. S. Rees, I. Pop, Local thermal non-equilibrium in porous medium Convection, in: Transport Phenomena in Porous Media III, D. B. Ingham, I. Pop (Eds.), Pergamon, 2005, pp. 147.173. 15. P. Vadasz, Turbulence in porous media, in Handbook of Porous Media (Ed. K. Vafai), Marcel
Dekker, 2000, pp. 679-754.

16. Y. Kakimoto, P. G. Siddheshwar, T. Masuoka, Nonlinear instability of thermal convection in anisotropic porous media, Proc. $39^{\text {th }}$ National Heat Transfer Symposium of Japan, Japan Heat Tronsfer Society, 2003, pp. 795-796.

17. N. Rudraiah, P. G. Siddheshwar, T. Masuoka, Nonlinear convection in porous media: A review, J. Porous Media, vol. 6, 2003, pp. 1-32.

18. A. Mojtabi, M-C Charrier-Mojtabi, Double-diffusive convection in porous media, in Handbook of Porous Media (Ed. K. Vafai), Marcel Dekker, 2000, pp. 559-603. 